\title{
Efeito de Exercícios de Pilates na Postura de Portadores de HAM/TSP Associado ao HTLV-1*
}

\author{
Diana Ferreira Livramento ${ }^{1}$ \\ Acadêmica do $4^{\circ}$ ano do curso de Graduação \\ em Fisioterapia da Escola Bahiana de \\ Medicina e Saúde Pública \\ Luma Souza Duran \\ Acadêmica do $4^{\circ}$ ano do curso de Graduação \\ em Fisioterapia da Escola Bahiana de \\ Medicina e Saúde Pública \\ Médico, Livre Docente, Professor Titular e \\ Coordenador do Centro de Referência \\ Bernardo Galvão-Castro \\ Multidisciplinar de Pesquisa e Assistência ao \\ paciente com HTLV-1 da Escola Bahiana de \\ Medicina e Saúde Pública \\ Fisioterapeuta, Doutora, Professora Adjunta e \\ Kátia Nunes Sá \\ Líder do Grupo de Pesquisa em Dinâmica do \\ Sistema Neuromusculoesquelético da Escola \\ Bahiana de Medicina e Saúde Pública \\ Tipo de Pesquisa \\ Artigo Original \\ Trabalho de Conclusão de Curso de \\ Origem da Pesquisa \\ Graduação em Fisioterapia da Escola Bahiana \\ de Medicina e Saúde Pública das duas \\ primeiras autoras. \\ Tipo de Análise do Manuscrito \\ Triple Blind Peer Review \\ Recebido em Dez/ 2011 \\ Aprovado em Dez/ 2011
}

\section{RESUMO}

Introdução: 0 HTLV-1 é um retrovírus que infecta o sistema imunológico e pode causar a paraparesia espástica tropical (HAM/TSP), doença neurológica que gera importantes alterações biomecânicas e têm como conseqüência acometimentos posturais. Exercícios específicos como propostos pelo Método Pilates podem fortalecer músculos, melhorar a flexibilidade e desordens posturais o que pode melhor a funcionalidade desses pacientes. Objetivo: Verificar o efeito de exercícios do Método Pilates nas alterações posturais em pacientes com HAM/TSP. Metodologia: Ensaio clínico randomizado em pacientes com HAM/TSP de um Centro de Referência em HTLV na cidade de Salvador. Foi realizada avaliação postural com fotografias digitais aplicando-se o protocolo SAPO versão 0,67 antes e após a intervenção. Os exercícios de um programa de Pilates foram realizados por três meses consecutivos em grupos de quatro participantes cada. Os dados foram analisados pelo programa SPSS versão 14.0, com significância de 5\%. Resultados: Participaram do estudo 14 indivíduos sorteados para serem incluídos 7 no grupo teste e 7 no grupo controle. A idade variou de 33 a 55 anos e a maioria era do sexo feminino (64,3\%). Os ângulos/alinhamentos posturais com maiores desvios foram alinhamento horizontal da EIAS, alinhamento vertical do tronco, alinhamento horizontal da cabeça, ângulo Q esquerdo, ângulo do quadril, ângulo do joelho e o ângulo do tornozelo. Conclusão: Exercícios do método Pilates promoveram efeitos positivos em relação ao alinhamento postural nesses indivíduos, sugerindo que esta alternativa de intervenção pode contribuir com a melhora de uma das principais causas de dor mecânica nestes pacientes.

Palavras-chave: HTLV; HAM/TSP; Postura; Pilates

${ }^{1}$ Agradecemos à acadêmica Tatiana Simões pela colaboração na coleta e análise dos dados. 


\section{Effects of Pilates Exercises Posture in Patients with HAM / TSP HTLV-1 *Associated}

\section{ABSTRACT}

Background: The HTLV-1 is a retrovirus that infects the immune system and can cause tropical spastic paraparesis (HAM / TSP), neurological disease which generates significant biomechanical changes and have consequently postural bouts. Specific exercises as proposed by Pilates can strengthen muscles, improve flexibility and postural disorders which can better the functionality of these patients. Objective: To investigate the effect of Pilates exercise on postural changes in patients with HAM / TSP. Methods: Randomized clinical trial in patients with HAM / TSP in an HTLV Reference Center in Salvador. Postural evaluation was performed with digital photographs applying the protocol SAPO version 0.67 before and after the intervention. The exercise of a Pilates program were conducted for three consecutive months in groups of four participants each. Data were analyzed using SPSS 14.0, with a significance of 5\%. Results: The study included 14 subjects selected for inclusion in the test group 7 and 7 in the control group. The age ranged from 33 to 55 years and most were female $(64.3 \%)$. The angles / postural alignment with larger deviations were horizontal alignment of the ASIS, the trunk vertical alignment, horizontal alignment of the head, left $Q$ angle, hip angle, knee angle and ankle angle. Conclusion: Pilates Exercises promoted positive effects in relation to postural alignment in these individuals, suggesting that this alternative intervention can contribute to the improvement of a major cause of mechanical pain in these patients.

Keywords: HTLV; HAM / TSP; Posture; Pilates

\section{Efectos de Pilates ejercicios de postura en pacientes con HAM / TSP HTLV-1* Asociada}

\section{RESUMEN}

Antecedentes: El HTLV-1 es un retrovirus que infecta el sistema inmunológico y puede causar la paraparesia espástica tropical (HAM / TSP), enfermedad neurológica que genera importantes cambios biomecánicos y tener peleas por consiguiente, la postura. Los ejercicios específicos propuestos por Pilates puede fortalecer los músculos, mejorar la flexibilidad y los trastornos posturales que pueden mejorar la funcionalidad de estos pacientes. Objetivo: Investigar el efecto del ejercicio de Pilates en los cambios posturales en pacientes con HAM / TSP. Métodos: ensayo clínico aleatorizado en pacientes con HAM / TSP en un Centro de Referencia de HTLV en Salvador. Evaluación postural se realizó con las fotografías digitales que aplican el protocolo de la versión SAPO 0,67 antes y después de la intervención. El ejercicio de un programa de Pilates se llevaron a cabo durante tres meses consecutivos en grupos de cuatro participantes cada uno. Los datos fueron analizados utilizando el programa SPSS 14.0, con una significación del 5\%. Resultados: El estudio incluyó a 14 sujetos seleccionados para su inclusión en el grupo de prueba de 7 y 7 en el grupo control. La edad varió de 33 a 55 años y la mayoría eran mujeres (64,3\%). Los ángulos de la alineación postural o con mayores desviaciones fueron la alineación horizontal de la EIAS, la alineación del tronco vertical, la alineación horizontal de la cabeza, dejó ángulo Q, ángulo de la cadera, ángulo de la rodilla y el ángulo del tobillo. Conclusión: Ejercicios Pilates promovidas efectos positivos en relación con la alineación postural en estos individuos, lo que sugiere que esta alternativa de intervención puede contribuir a la mejora de una de las principales causas de dolor mecánico en estos pacientes.

Palabras clave: HTLV; HAM / TSP, Postura, Pilates 


\section{Effets de la méthode Pilates Exercices posture chez les patients atteints HAM / TSP HTLV-1* Associated}

\section{RÉSUMÉ}

Contexte: Le HTLV-1 est un rétrovirus qui infecte le système immunitaire et peuvent causer la paraparésie spastique tropicale (HAM / TSP), maladie neurologique qui génère d'importants changements biomécaniques et ont par conséquent des épisodes posturaux. Des exercices spécifiques tels que proposés par la méthode Pilates peut renforcer les muscles, améliorer les troubles de flexibilité et postural qui peut mieux les fonctionnalités de ces patients. Objectif: étudier l'effet de l'exercice Pilates sur les changements posturaux chez les patients atteints HAM / TSP. Méthodes: Essai clinique randomisé chez des patients atteints HAM / TSP dans un Centre de référence HTLV à Salvador. Évaluation posturale a été réalisée avec des photographies numériques en appliquant la version de protocole de SAPO 0,67 avant et après l'intervention. L'exercice d'un programme de Pilates ont été menées pendant trois mois consécutifs dans les groupes de quatre participants chacune. Les données ont été analysées à l'aide de SPSS 14.0, avec une signification de 5\%. Résultats: L'étude a inclus 14 sujets sélectionnés pour l'inclusion dans le groupe test 7 et 7 dans le groupe témoin. L'âge variait de 33 à 55 ans et plus étaient des femmes (64,3\%). Les angles / alignement postural avec de grandes déviations étaient alignement horizontal de l'ASIS, l'alignement tronc vertical, alignement horizontal de la tête, à gauche angle $Q$, angle de la hanche, du genou et l'angle de l'angle de cheville. Conclusion: Pilates Exercices promus effets positifs en ce qui concerne l'alignement postural chez ces individus, ce qui suggère que cette intervention alternative peut contribuer à l'amélioration d'une cause majeure de douleurs mécaniques chez ces patients.

Mots-clés: HTLV; HAM / TSP; Posture; Pilates

\section{Introdução}

0 vírus linfotrópico para células T humanas - HTLV-1 é um vírus que afeta principalmente os linfócitos T do organismo sendo que sua ação no corpo humano é lenta, considerado de início tardio.1,2 A transmissão do HTLV-1 se dar por contato sexual, uso de drogas injetáveis e verticalmente de mãe para filho.3 Estimativas apontam que o Brasil é o país com o maior número absoluto de casos e Salvador foi considerada a cidade brasileira com a maior prevalência dessa infecção, estimada em 1,76\%, cerca de 50 mil pessoas.1, 4

Este vírus está relacionado principalmente com uma doença neurológica crônica incapacitante, a paraparesia espástica tropical/mielopatia associada ao HTLV-1 (HAM/TSP). 5 Geralmente o quadro clínico da HAM/TSP é lentamente progressivo sendo que suas principais conseqüências biomecânicas e funcionais são bexiga neurogênica, fadiga ao caminhar resultante da espasticidade em membros inferiores, fraqueza muscular em cintura pélvica e membros inferiores, encurtamento muscular, hipomobilidade articular e dor lombar resultando em acometimentos posturais. 6

Postura é a relação cinética e cinemática entre as estruturas do corpo humano. Quando ossos, músculos e articulações se encontram em equilíbrio dinâmico eles geram mínimos esforços e sobrecarga ao corpo caracterizando assim um alinhamento esquelético ideal. Quando essas estruturas se encontram desequilibradas como por exemplo na fraqueza muscular, em desordens na propriocepção e amplitude de movimento há uma interferência na capacidade de manutenção do centro de gravidade do indivíduo dentro de sua base de sustentação, alterando assim seu alinhamento postural.7

O Método Pilates, tem como base um conceito denominado de contrologia, definido por Joseph Pilates como o controle consciente dos movimentos musculares do corpo, no qual seus 
princípios são concentração, consciência, controle, "centramento, respiração e movimento harmônico. Os exercícios na maioria das vezes, são realizados na posição deitada, havendo diminuição dos impactos nas articulações de sustentação do corpo e, principalmente, na coluna vertebral, permitindo recuperação das estruturas musculares, articulares e ligamentares.8

A técnica é baseada no centro de força (Power House) com enfoque na ativação de músculos específicos, como abdominais, glúteos e paravertebrais no qual a fixação proximal seja bem estabilizada e consequentemente o movimento distal seja forte e eficiente.9,10

0 treinamento com o Pilates tem como objetivo a melhora da flexibilidade geral do corpo, busca da saúde através do fortalecimento do "centro de força", melhora da postura e coordenação da respiração com os movimentos realizados, sendo indicado para o tratamento de diversos pacientes, incluindo pacientes neurológicos .11

Cooper et al em 2009 afirmam que ainda não há um consenso sobre o tratamento neurológico dessa patologia, destacando a imensa importância da fisioterapia motora e urinária para esses indivíduos.

Observando a grande prevalência de pessoas com HAM/TSP em Salvador, a utilização de novos programas de exercícios específicos, como o Método Pilates, que busquem fortalecimento muscular, melhora da flexibilidade, mobilidade articular e das desordens posturais serão de grande importância para a melhora da qualidade de vida desses pacientes de forma preventiva e terapêutica.

0 objetivo geral deste estudo é verificar o efeito do Método Pilates nas alterações posturais em pacientes com HAM/TSP.

\section{Material \& Método}

0 estudo realizado é um ensaio clínico randomizado, de corte transversal, duplo-cego cuja amostra foi composta por pacientes com HAM/TSP de um Centro de referência de HTLV da cidade de Salvador.

Foram incluídos no estudo os pacientes matriculados no Centro Integrativo e Multidisciplinar de HTLV, pertencentes a ambos os sexos, portadores de HAM-TSP classificado no grupo Definido, com lombalgia há pelo menos seis meses, com idade igual ou superior a 20 anos e igual ou inferior a 59 anos, com nível cognitivo preservado, com condições de realizar o esvaziamento vesical antes de cada sessão de Pilates voluntariamente ou através de cateterismo e concordarem em participar do estudo mediante assinatura do Termo de Consentimento Livre e Esclarecido (TCLE).

Foram excluídos deste, portadores de deformidade física, déficits neurológicos associados desvinculados à patologia viral, pacientes que estavam realizando outra atividade física e gestantes.

De acordo com o cálculo amostral, realizado pela calculadora on-line do Laboratório de Epidemiologia e Estatística da Universidade São Paulo (LEE), considerando prevalência de 5\% de pacientes com HAM/TSP dos 1.000 matriculados no CHTLV, uma precisão absoluta de 5\% e nível de significância de $5 \%$ seriam necessários 12 pacientes no grupo teste e 12 pacientes no grupo controle para responder à pergunta de investigação.

Foram formados 4 grupos, 2 grupo testes e 2 grupos controles. Em cada grupo teste foram alocados 4 pacientes, totalizando 8 indivíduos nesse grupo. No grupo controle foram alocados 3 pacientes em cada grupo. No total de 6 participantes. A formação dos grupos foi feita através de sorteio por um membro da equipe cego.

Os participantes foram fotografados de corpo inteiro nas vistas anterior, lateral direita, lateral esquerda e posterior. A primeira avaliação foi realizada em janeiro de 2010 no qual foram submetidos a uma avaliação postural ortostática bi-dimensional utilizando o SAPO® 
versão 0.67 de acordo com o protocolo determinado pelo sistema, disponível em http:// sapo.iv.org.br/portal. Para essa avaliação foi utilizada a câmera digital (Olympus Imaging Corporation D-535, Made in China, com resolução de 3.2 megapixels), fixadas semiesferas de isopor em pontos anatômicos pré-estabelecidos pelo protocolo, estes posicionados por uma equipe previamente treinada. A re-avaliação foi realizada três meses após a primeira avaliação na qual foi aplicado o mesmo procedimento.

As mulheres do estudo foram orientadas a utilizarem short e sutiã e os homens apenas short para uma melhor visualização dos segmentos corporais.

Os selecionados para o grupo teste assistiram a uma aula explicativa sobre o método Pilates e posteriormente foram submetidos a um programa de exercícios baseados nesse método por 3 meses por equipe previamente treinada, de forma padronizada.

0 protocolo de exercícios (disponível em www.bahiana.edu.br/pilates) utilizando os equipamentos Reformer e Cadillac foi implementado durante 30 sessões, com freqüência de duas sessões semanais, cada uma com duração de uma hora. As sessões foram ministradas por um instrutor com 15 anos de formação no Método Pilates e mais um assistente.

Observando que a população do estudo apresenta acometimentos predominantes em membros inferiores, as medidas foram focadas nessa região, não descartando a influência dos desvios em outros segmentos do corpo. 0 estudo apresenta como variável independente, o protocolo de exercícios do Método Pilates e, como variável dependente, as alterações posturais segundo as seguintes variáveis: 1) Alinhamento horizontal da cabeça no qual verifica inclinação cervical; 2) Alinhamento horizontal das EIAS verificando inclinação pélvica; 3) Ângulo frontal do membro inferior direito/ esquerdo e possível tendência a geno varo e valgo; 4) Ângulo Q direito/esquerdo; 5) Ângulo perna/retropé direito/esquerdo no qual verifica o alinhamento do calcâneo; 6) Alinhamento vertical da cabeça verifica o deslocamento anterior e posterior da cabeça; 7) Alinhamento vertical do tronco no qual verifica possível deslocamento anterior e posterior do tronco; 8) Ângulo do quadril verificando flexão e extensão dessa articulação; 9) Alinhamento horizontal da pelve verifica possível anteversão e retroversão; 10) Ângulo do joelho verifica flexão e extensão; 11) Ângulo do tornozelo.

Os dados foram tabulados e analisados pelo software SSPS versão 14.0 for Windows, considerando um nível de significância de 5\%. Foram utilizados os testes estatísticos t de Student pareado para análise intra-grupos e teste de Mann-Whitney para análise inter-grupos.

0 estudo foi aprovado pelo Comitê de Ética em Pesquisa da Escola Bahiana de Medicina e Saúde Pública em Agosto de 2010 sob o protocolo 083/2012 em concordância com o Conselho Nacional de Ética em Pesquisa Resolução 196/96. Os pacientes que aceitaram participar do estudo voluntariamente assinaram o Termo de Consentimento Livre e Esclarecido.

\section{Resultados}

A amostra inicialmente foi constituída de 15 portadores de mielopatia associada ao vírus HTLV-1, sendo que um indivíduo foi excluído devido à ausência nas atividades, totalizando 14 participantes no final do estudo. No grupo Teste $(\mathrm{N}=8), 6(75 \%)$ são pertecentes ao sexo feminino, com média de idade de 43,5 \pm 7,4 anos e apresentando IMC normal em 4 indivíduos (50\%) e 4 (50\%) com sobrepeso.

Nota-se que o nível de escolaridade varia de baixo a médio. Observa-se que 4 (50,0\%) indivíduos são casados e 5 (62.5\%) aposentados por invalidez ou por idade. Grande parte já realizou tratamento fisioterapêutico $7(87.5 \%)$, porém nenhum é praticante de exercício físico. Relativo a utilização de dispositivos auxiliares 4 (50\%) não os usa entretanto 3 (37.5\%) necessita de um par de muletas.

Já no grupo Controle $(\mathrm{N}=6)$ há uma igualdade na quantidade de homens e mulheres, com média de idade de 50,8 $\pm 5,26$ anos, com IMC normal 3(50\%), e a maioria apresentando um nível de escolaridade alto 3(50\%). Em relação ao estado civil grande parte támbem é casado $3(50 \%)$, aposentados $4(66,7 \%)$, já realizaram fisioterapia $5(83,3 \%)$ e são sedentários 
5(83\%). Grande parte dos indivíduos desse grupo também não utilizam nenhum dispositivo auxiliar 5 (83,3\%) (Tabela 1$)$.

Tabela 1

Caracterização sociodemográfica e clínica da amostra de 14 portadores de HTLV associado ao HAM/TSP de um Centro de Referência em HTLV, em Salvador/Ba

\begin{tabular}{|c|c|c|}
\hline Variável & $\begin{array}{c}\text { Grupo Teste }(\mathrm{N}=8) \\
\mathrm{N}(\%)\end{array}$ & $\begin{array}{c}\text { Grupo Controle }(N=6) \\
N(\%)\end{array}$ \\
\hline \multicolumn{3}{|l|}{ Sexo } \\
\hline Feminino & $6(75 \%)$ & $3(50 \%)$ \\
\hline Masculino & $2(25 \%)$ & $3(50 \%)$ \\
\hline \multicolumn{3}{|l|}{ IMC } \\
\hline Eutrófico & $4(50 \%)$ & $3(50 \%)$ \\
\hline Sobrepeso & $4(50 \%)$ & $2(33,3 \%)$ \\
\hline Obesidade Grau I & 0 & $1(16,7 \%)$ \\
\hline \multicolumn{3}{|l|}{ Escolaridade } \\
\hline 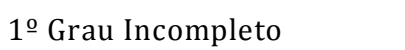 & $3(37,5 \%)$ & $1(16,7 \%)$ \\
\hline 1 Grau Completo & $1(12,5 \%)$ & $1(16,7 \%)$ \\
\hline 2으 Grau Incompleto & $3(37,5 \%)$ & $1(16,7 \%)$ \\
\hline 2o Grau Completo & $1(12,5 \%)$ & $3(50 \%)$ \\
\hline \multicolumn{3}{|l|}{ Estado Civil } \\
\hline Solteiro & $2(25 \%)$ & $1(16,7 \%)$ \\
\hline Casado & $4(50 \%)$ & $3(50 \%)$ \\
\hline Divorciado & $2(25 \%)$ & $1(16,7 \%)$ \\
\hline Viúvo & 0 & $1(16,7 \%)$ \\
\hline \multicolumn{3}{|l|}{ Ocupação } \\
\hline Aposentado & $5(62,5 \%)$ & $4(66,7 \%)$ \\
\hline Ativo & $3(37,5 \%)$ & $2(33,3 \%)$ \\
\hline \multicolumn{3}{|l|}{ Dispositivo Auxiliar } \\
\hline Nenhum & $4(50 \%)$ & $5(83,3 \%)$ \\
\hline Uma muleta & $1(12,5 \%)$ & 0 \\
\hline Par de muletas & $3(37,5)$ & $1(16,7 \%)$ \\
\hline \multicolumn{3}{|l|}{ Tratamentos anteriores } \\
\hline Nenhum & 0 & $1(16,7 \%)$ \\
\hline Fisioterapia & $7(87,5 \%)$ & $5(83,3 \%)$ \\
\hline Fisioterapia e Hidroterapia & $1(12,5 \%)$ & 0 \\
\hline \multicolumn{3}{|l|}{ Exercício Físico } \\
\hline Ativo & 0 & $1(16,7 \%)$ \\
\hline Sedentário & $8(100 \%)$ & $5(83,3 \%)$ \\
\hline
\end{tabular}

Foram observadas diferenças na postura dos indivíduos antes e após a intervenção através do protocolo de exercícios do método Pilates aplicado. Considerando a diferença dos ângulos posturais dos 14 indivíduos, foi observada significância estatística em algumas das variáveis correlacionadas como redução do ângulo frontal do membro inferior direito $(p<0,01)$. Na vista lateral direita houve melhora no alinhamento vertical do corpo $(p=0,05)$ mostrando melhor alinhamento do indivíduo na posição ereta, como também no alinhamento vertical do tronco $(p=0,01)$, este observado também significância na vista lateral esquerda 
$(\mathrm{p}<0,01)$. Nesta vista ainda observou-se um melhor alinhamento vertical da cabeça $(\mathrm{p}<0,01)$, do ângulo do joelho $(\mathrm{p}=0,02)$ e do ângulo do tornozelo $(\mathrm{p}=0,02)$. (Tabela 2)

Tabela 2

Média das diferenças dos ângulos dos 14 portadores de HTLV associado ao HAM/TSP de um Centro de Referência em HTLV , em Salvador/BA

\begin{tabular}{|c|c|c|c|c|}
\hline Variáveis & Média & DP & IC $95 \%$ & $P=0,05$ \\
\hline $\mathrm{AHC}$ & 0,40 & 4,34 & $-2,10_{-} 2,90$ & 0,73 \\
\hline AHEIAS & $-0,18$ & 3,00 & $-1,922_{-} 1,55$ & 0,81 \\
\hline AFMID & $-3,49$ & 3,59 & $-5,57 \ldots-1,41$ & $<0,01$ \\
\hline AFMIE & $-2,22$ & 4,25 & $-4,68 \_0,22$ & 0,07 \\
\hline AQD & 0,49 & 7,89 & $-4,06 \_5,05$ & 0,81 \\
\hline$A Q E$ & $-2,21$ & 8,71 & $-7,24 \ldots 2,81$ & 0,35 \\
\hline APRD & $-0,58$ & 8,06 & $-5,23 \_4,06$ & 0,79 \\
\hline AFMIE & $-2,22$ & 4,25 & $-4,68 \_0,22$ & 0,07 \\
\hline$A Q D$ & 0,49 & 7,89 & $-4,066_{-} 5,05$ & 0,81 \\
\hline AQE & $-2,21$ & 8,71 & $-7,24 \ldots 2,81$ & 0,35 \\
\hline APRD & $-0,58$ & 8,06 & $-5,23 \_4,06$ & 0,79 \\
\hline APRE & 1,25 & 5,34 & $-1,83 \ldots 4,33$ & 0,39 \\
\hline AVCLD & 6,00 & 11,31 & $-0,53 \_12,53$ & 0,06 \\
\hline AVTLD & $-2,15$ & 2,90 & $-3,822_{-}-0,47$ & 0,01 \\
\hline AQLD & $<0,01$ & 5,46 & $-3,14 \_3,16$ & 0,99 \\
\hline AHPLD & $-0,75$ & 6,02 & $-4,22 \_2,72$ & 0,64 \\
\hline AJLD & 1,52 & 9,45 & $-3,93 \_6,98$ & 0,55 \\
\hline ATLD & $-1,35$ & 4,09 & $-3,71{ }_{-} 1,01$ & 0,23 \\
\hline AVCOLD & $-0,75$ & 1,35 & $-1,54 \_0,02$ & 0,05 \\
\hline AVCLE & 8,81 & 10,65 & $2,66{ }_{-} 14,96$ & $<0,01$ \\
\hline AVTLE & $-2,35$ & 2,53 & $-3,82{ }_{-}-0,89$ & $<0,01$ \\
\hline AQLE & $-1,80$ & 5,65 & $-5,06 \_1,46$ & 0,25 \\
\hline AHPLE & $-2,27$ & 7,80 & $-6,77 \ldots 2,23$ & 0,29 \\
\hline AJLE & 5,02 & 7,34 & $0,78 \_9,26$ & 0,02 \\
\hline ATLE & $-3,88$ & 5,91 & $-7,30_{-}-2,45$ & 0,02 \\
\hline AVCOLE & $-0,67$ & 3,18 & $-2,50 \_1,16$ & 0,44 \\
\hline
\end{tabular}

LEGENDA: AHC: Alinhamento horizontal da cabeça, AHEIAS: Alinhamento horizontal das espinhas ilíacas ântero-superiores, AFMID: Ângulo frontal do membro inferior direito, AFMIE: Ângulo frontal do membro inferior esquerdo, AQD: Ângulo Q direito, AQE: Ângulo Q esquerdo, APRD: Ângulo perna/retropé direito, APRE: Ângulo perna/retropé esquerdo, AVCLD: Alinhamento vertical da cabeça (Vista Lateral Direita VLD), AVTLD: Alinhamento vertical do tronco (VLD), AQLD: Ângulo do quadril (VLD), AHPLD: Alinhamento horizontal da pélvis (VLD), AJLD: Ângulo do joelho (VLD), ATLD: Ângulo do tornozelo (VLD), AVCOLD: Alinhamento vertical do corpo (VLD), AVCLE: Alinhamento vertical da cabeça Vista lateral Esquerda (VLE), AVTLE: Alinhamento vertical do tronco (VLE), AQLE: Ângulo do quadril (VLE), AHPLE: Alinhamento horizontal da pélvis (VLE), AJLE: : Ângulo do joelho (VLE), ATLE: Ângulo do tornozelo (VLE), AVCLE: : Alinhamento vertical do corpo (VLE).

OBS: *Teste t de Student Pareado 
No grupo Teste foi observada uma significância estátistica vista após a intervenção do Pilates no ângulo frontal do membro inferior direito $(p=0,05)$, alinhamento vertical da cabeça tanto na vista lateral direita $(p=0,02)$ quanto na lateral esquerda $(p=0,04)$ (Figura 1) no alinhamento vertical do tronco também na vista lateral direita $(\mathrm{p}=0,01)$ bem como na vista lateral esquerda $(\mathrm{p}=0,02)$ (Figura 2) e o ângulo do joelho na vista lateral esquerda $(p=0,05)$.

Enquanto que no grupo controle como já esperado não foi observada significância estatística na grande maioria das variáveis correlacionadas, apenas o ângulo frontal do membro inferior direito $(\mathrm{p}=0,03)$ (Tabela 3$)$.

Tabela 3

Comparação dos ângulos posturais do Grupo Teste e Controle antes e após a intervenção de exercícios de Pilates da amostra de 14 portadores de HTLV associado ao HAM/TSP de um Centro de Referência em HTLV, em Salvador/BA

\begin{tabular}{|c|c|c|c|c|}
\hline Variáveis & $\begin{array}{c}\text { Grupo Teste } \\
\text { (N=8) } \\
\text { Média } \\
\text { (IC 95\%) }\end{array}$ & $P=0,05$ & $\begin{array}{c}\text { Grupo Controle } \\
(\mathrm{N}=6) \\
\text { Média } \\
\text { (IC } 95 \%)\end{array}$ & $P=0,05$ \\
\hline $\mathrm{AHC}$ & $1,48\left(-2,69 \_5,67\right)$ & 0,42 & $-1,01\left(-4,29 \_2,19\right)$ & 0,44 \\
\hline AHEIAS & $-1,21\left(-3,83 \_1,41\right)$ & 0,31 & $1,18\left(-1,34 \_3,70\right)$ & 0,28 \\
\hline AFMID & $-3,42\left(-6,96 \_0,11\right)$ & 0,05 & $-3,58\left(-6,66_{-}-0,50\right)$ & 0,03 \\
\hline AFMIE & $-2,53\left(-6,69 \_1,61\right)$ & 0,19 & $-1,81\left(-5,47 \_1,83\right)$ & 0,25 \\
\hline AQD & $0,91\left(-6,80 \_8,63\right)$ & 0,78 & $-0,06\left(-6,86 \_6,73\right)$ & 0,98 \\
\hline AQE & $0,41\left(-7,64 \_8,46\right)$ & 0,97 & $-5,71\left(-12,49 \_1,06\right)$ & 0,08 \\
\hline APRD & $-0,72\left(-7,17 \_5,72\right)$ & 0,79 & $-0,40\left(-10,09 \_9,29\right)$ & 0,92 \\
\hline APRE & $1,82\left(-2,37 \_6,02\right)$ & 0,33 & $0,48\left(-5,95 \_6,92\right)$ & 0,85 \\
\hline AVCLD & $11,16\left(1,53 \_20,79\right)$ & 0,02 & $-0,88\left(-8,13 \_6,37\right)$ & 0,76 \\
\hline AVTLD & $-2,45\left(-4,266_{-}-0,63\right)$ & 0,01 & $-1,75\left(-5,82 \_2,32\right)$ & 0,31 \\
\hline AQLD & $0,15\left(-3,99 \_4,29\right)$ & 0,93 & $-0,18\left(-7,08 \_6,71\right)$ & 0,94 \\
\hline AHPLD & $-2,26\left(-5,57 \_1,04\right)$ & 0,15 & $1,26\left(-7,11_{-} 9,64\right)$ & 0,71 \\
\hline AJLD & $3,25\left(-4,55 \_11,05\right)$ & 0,35 & $-0,78\left(-11,24 \_9,68\right)$ & 0,85 \\
\hline ATLD & $-0,85\left(-4,85_{-}-0,50\right)$ & 0,63 & $-2,01\left(-5,40 \_1,37\right)$ & 0,18 \\
\hline AVCOLD & $-1,03\left(-2,19 \_-2,12\right)$ & 0,07 & $-0,38\left(-1,79 \_1,03\right)$ & 0,51 \\
\hline AVCLE & $10,87\left(0,07 \_21,67\right)$ & 0,04 & $6,06\left(-1,01 \_13,15\right)$ & 0,07 \\
\hline AVTLE & $-2,66\left(-4,84_{-}-0,47\right)$ & 0,02 & $-1,95\left(-4,68 \_0,78\right)$ & 0,12 \\
\hline AQLE & $-0,45\left(-4,44{ }_{-} 3,54\right)$ & 0,79 & $-3,60\left(-10,57 \_3,37\right)$ & 0,24 \\
\hline AHPLE & $-1,93\left(-8,54 \_4,66\right)$ & 0,51 & $-2,71\left(-11,51_{-}-0,79\right)$ & 0,46 \\
\hline AJLE & $6,38\left(-0,26 \_13,04\right)$ & 0,05 & $3,21\left(-3,79 \_10,22\right)$ & 0,29 \\
\hline ATLE & $-3,15\left(-8,09 \_1,79\right)$ & 0,17 & $-4,86\left(-11,50 \_1,79\right)$ & 0,11 \\
\hline AVCOLE & $-2,06\left(-4,88 \_0,76\right)$ & 0,12 & $1,18\left(-0,66 \_3,03\right)$ & 0,16 \\
\hline
\end{tabular}

LEGENDA: AHC: Alinhamento horizontal da cabeça, AHEIAS: Alinhamento horizontal das espinhas ilíacas ântero-superiores, AFMID: Ângulo frontal do membro inferior direito, AFMIE: Ângulo frontal do membro inferior esquerdo, AQD: Ângulo Q direito, AQE: Ângulo Q esquerdo, APRD: Ângulo perna/retropé direito, APRE:Ângulo perna/retropé esquerdo, AVCLD: Alinhamento vertical da cabeça (Vista Lateral Direita VLD), AVTLD: Alinhamento vertical do tronco (VLD), AQLD: Ângulo do quadril (VLD), AHPLD: Alinhamento horizontal da pélvis (VLD), AJLD: Ângulo do joelho (VLD), ATLD: Ângulo do tornozelo (VLD), AVCOLD: Alinhamento vertical do corpo (VLD), AVCLE: Alinhamento vertical da cabeça Vista lateral Esquerda (VLE), AVTLE: Alinhamento vertical do tronco (VLE), AQLE: Ângulo do quadril (VLE), AHPLE: Alinhamento horizontal da pélvis (VLE), AJLE: : Ângulo do joelho (VLE), ATLE: Ângulo do tornozelo (VLE), AVCLE: : Alinhamento vertical do corpo (VLE). 
OBS: *Teste t de Student Pareado
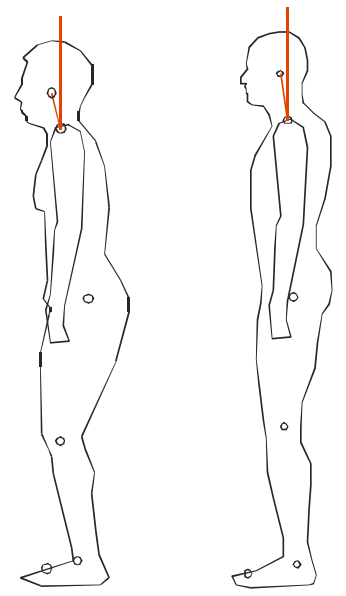

Figura 1: Alinhamento vertical da cabeça Vista Esquerda
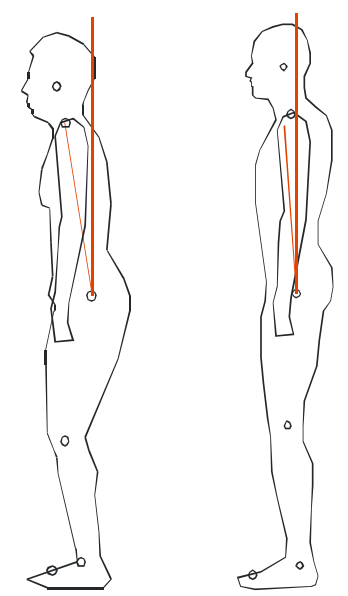

Figura 2: Alinhamento vertical do tronco Vista Lateral Esquerda

\section{Discussão}

0 presente estudo que buscou verificar o efeito de uma intervenção através de exercícios do método Pilates na postura de portadores de HAM/TSP associada ao HTLV-1, encontrou impacto positivo na população estudada. Embora se verifique escassez de pesquisas sobre o tratamento funcional para indivíduos com HAM/TSP, ensaios clínicos utilizando este método têm sido desenvolvidos com bons resultados em outros pacientes. $9,10,11$

É consenso na literatura que o Método Pilates preconiza a melhora das relações musculares agonista e antagonista, gerando um fortalecimento global da cadeia muscular e não de um músculo isolado. Isso gera um alinhamento dos segmentos corporais repercutindo positivamente na postura do indivíduo.13,14,15

Avaliando os efeitos dos exercícios de Pilates na postura dos pacientes com HAM/TSP desse estudo, observou-se uma melhora significativa em parte das variáveis correlacionadas, mostrando assim que os participantes do Grupo Teste obtiveram um melhor alinhamento cervical e de tronco reduzindo deslocamentos anteriores tanto nas vistas laterais direita e esquerda, diminuição do varismo de joelho direito e uma diminuição do geno flexo de joelho. Corroborando com este resultado, estudos prévios14,15 têm verificado efeito corretivo no alinhamento postural de várias partes do corpo dos participantes sem uma padronização. Porém, estas variações podem ser explicadas pelo reduzido número de participantes com diferentes quadros clínicos crônicos.

0 protocolo de avaliação do sistema de fotogrametria também tem sua aplicação em fases iniciais, 22 necessitando de maior validação em diferentes populações e com diferentes avaliadores para que se possam observar mudanças em mensurações angulares significativas. Outros estudos têm referido pouca precisão para os resultados com pequenas diferenças observadas com exercícios de Pilates por ainda não termos uma amostra de estudos realizados por avaliadores experientes. 150 detalhamento do protocolo de exercícios e o desenvolvimento das habilidades na mensuração dos resultados podem justificar o baixo nível de evidência no assunto. Porém, a avaliação postural através do Software de avaliação 
postural (SAPO) tem sido bem aceita e precisa nos trabalhos científicos, possuindo fidedignidade com resultados quando avaliada postura dos indivíduos.22

Um dos fatores mais influentes no trabalho da postura são os exercícios de fortalecimento dos músculos posturais. Quando o centro de força está fortalecido, o indivíduo consegue manter o alinhamento dos segmentos corporais.14,17 0 músculo postural multífido tem sua função relacionada à extensão de tronco. Tem-se demonstrado que sua função está prejudicada em pacientes com lombalgia e em pacientes neurológicos.11 Exercícios de extensão de tronco, contrações de transverso abdominal e musculatura perineal são utilizados para fortalecer o centro de força tem constatado com medidas quantitativas o ganho da força.8

Pacientes com escoliose têm igualmente se beneficiados pelo método Pilates que se mostra eficiente em promover um equilíbrio entre flexores e extensores do tronco. 8,10 Essas relações de fortalecimento dos músculos posturais, principalmente do extensor da coluna após a intervenção do Pilates, podem explicar a melhora do alinhamento cervical e do tronco encontrados nos participantes com HAM/TSP desse estudo.

A posição da pelve influencia o alinhamento postural adequado ou não, esta deve estar em posição neutra.18,19 Os indivíduos da presente amostra apresentam um perfil neurológico característico com fraqueza em musculatura abdominal e glúteos, gerando flexão de quadril e instabilidade pélvica. Tem sido observado que quando há falta de sustentação da posição neutra da pelve, pode haver desalinhamentos em membros inferiores e ocorrer flexão de joelho para contrabalancear o deslocamento anterior do centro de massa. 19

A realização do movimento alinhada com o momento da expiração proposta pelo Pilates pode melhorar a estabilização da pelve. 19,20 Um trabalho de fortalecimento com a pelve em posição neutra durante os exercícios pode ser capaz de melhorar o alinhamento da pelve em conjunto com o fortalecimento de membros inferiores, 13 interferindo na melhora do alinhamento do joelho. 0 alinhamento horizontal da pelve não teve significância estatística nesse estudo, porém as alterações nos ângulos dos membros inferiores podem apontar para o ganho indireto induzido pelo exercício.

Um dos sistemas do corpo relacionado à postura e equilíbrio, é o sistema proprioceptivo. Como a perda da sensibilidade proprioceptiva pode ocorrer nesses indivíduos com HAM/TSP e ser mais um dos componentes que geram alterações posturais, 21 os efeitos benéficos dos exercícios de Pilates, geram também uma melhora na propriocepção tendo interferência nos efeitos positivos de alinhamento postural dos indivíduos da amostra. 14,15

Limitações nesse estudo foram verificadas em relação à pequena amostra e à dificuldade de cegamento dos pacientes e ao número reduzido de portadores de HTLV do grupo definido.

\section{Conclusão}

Exercícios do método Pilates promoveram efeitos positivos em relação ao alinhamento postural nesses indivíduos, sugerindo que esta alternativa de intervenção pode contribuir com a melhora de uma das principais causas de dor mecânica nestes pacientes.

\section{Referências}

1. DOURADO I et al. "HTLV-I in the general population of Salvador, Brazil: a city with African ethnic and sociodemographic characteristics", J Acquir Immune Defic Syndr, 34: 527-31. 2003.

2. SOARES Bcc, CASTRO Msm, PROIETTI Fa. “Epidemiologia do HTLV-I/II. In: Proietti AB, editor. HTLVI/ II", Belo Horizonte-MG: Fundação Centro de Hematologia e Hemoterapia de Minas Gerais, Cadernos Hemominas, XI: 53-75. 2000.

3. CATALAN-SOARES et al. "Os vírus linfotrópicos de células T humanos (HTLV) na última década (1990-2000): aspectos epidemiológicos”, Revista Brasileira de Epidemiologia, 4(2): 81-95. 2001. 
4. BITTENCOURT AL et al. "Human T-Cell lymphotropic virus type 1 infection among pregnant women in Northeastern Brazil”, J Acquir Immune Defic Syndr, 26: 490. 2001.

5. MORENO-CARVALHO O A, SANTOS JI, DI CREDICO G, GALVAO-CASTRO B. “Evidence of preferential female prevalence of HTLV-I associated tropical spastic paraparesis in Bahia-Brazil”, Arq Neuropsiquiatr, 50: 183-88. 1992.

6. ROMANELLI, Luiz C F; CARAMELLI, Paulo \& PROIETTI, Anna B F C. “O vírus linfotrópico de células T humanos tipo 1 (HTLV-1): Quando suspeitar da infecção?” Rev. Assoc. Med. Bras., 56(3): 340-47. 2010.

7. HAMILL J, KNUTZEN K. As bases Biomecânicas do Movimento Humano. São Paulo: Manole, 2002.

8. KOLYNIAK, I E G G; CAVALCANTI, Sonia M B; AOKI, Marcelo S. "Avaliação isocinética da musculatura envolvida na flexão e extensão do tronco: efeito do método Pilates®", São Paulo-SP: Revista Brasileira de Medicina do Esporte, 10(6): 487-90. Nov-Dez/ 04.

9. SOROSKY S, STILP S, AKUTHOTA V. "Yoga and pilates in the management of low back pain", Current Reviews in Musculoskeletal Medicine, 1: 39-47, 2008.

10. SILVA, Y. et al. "Análise da resistência externa e da atividade eletromiográfica do movimento de extensão de quadril realizado segundo o método Pilates", São Carlos-SP: Revista Brasileira de Fisioterapia, 13(1): 82-88. Jan-Fev/ 09.

11. BERTOLLA et al. "Efeito de um programa de treinamento utilizando o método Pilates $®$ na flexibilidade de atletas juvenis de futsal", Revista Brasileira de Medicina do Esporte, 13: 222-26, 2007.

12. COOPER SA, LOEFF Ms, TAYLOR Gp. “The neurology of HTLV-1 infection”, Pract Neurol, 9: 16-26. 2009.

13. HERRINGTON, L, Davies, R. "The influence of Pilates training on the ability to contract the transversus abdominis muscle in asymptomatic individuals", Journal of Bodywork and Movement Therapies, (9), 52-57. 2005.

14. NUNES Jr. et al. "Os efeitos do Método Pilates no alinhamento postural: estudo piloto", Rio de Janeiro-RJ: Fisioterapia Ser, 3(4): 210-15. Jul-Ago-Set/ 2008.

15. VIT, P P; LUCARELI, P; “Avaliação postural antes e após 75horas/aulas do Método Pilates em fisioterapeutas e educadores físicos", Centro Universitário São Camilo. Disponível em: <http:/ / www2.rc.unesp.br/ eventos/ educacao_fisica/ biomecanica2007/ upload/ 140-1-Acongresso\%20brasileiro\%20de\%20biomecanica.pdf>. (Acesso em: 18 de maio de 2011).

16. LATEY P. "Updating the principles of the Pilates method - part-2", Journal of Bodywork and Movement Therapies, 6(2): 94-101.2002.

17. BLUM, C L. "Chiropractic and Pilates therapy for the treatment of adult scoliosis", J Manipulative Physiol Ther, 25(E3): 2002.

18. KENDALL F P, MCCREARY E K, PROVANCE P G. Músculos - Provas e funções. (4th ed) São Paulo: Manole, 1995.

19. LEE D. A cintura pélvica: uma Abordagem para o exame e tratamento da região lombar, pélvica e do quadril. Barrueri: Manole; 2001, pp. 45-76.

20. APARICIO E; PÉREZ J. O autêntico método Pilates: a arte do controle. (Tradução Magda Lopes), São Paulo: Planeta do Brasil, 2005.

21. RIBAS, J G R; MELO, G C N. “Mielopatia associada ao vírus linfotrópico humano de células T do tipo 1 (HTLV-1)”, Rev Soc Bras Med Trop, 35(4): 377-84. 2002

22. SACCO, ICN et al. "Confiabilidade da fotogrametria em relação à goniometria para avaliação postural em membros inferiores”, São Carlos-SP: Revista Brasileira de Fisioterapia, 11(5): 411-17. SetOut/ 07. 